\title{
Optimizing Atrial Electrogram Classification Based on Local Ablation Outcome in Human Atrial Fibrillation
}

\author{
Arthur S Bezerra ${ }^{1}$; Takashi Yoneyama ${ }^{1}$; Diogo C Soriano ${ }^{2}$; Giorgio Luongo ${ }^{3}$; $\mathrm{Xin}^{4}{ }^{4}$; Flavia Ravelli ${ }^{5}$; Michela \\ Masè $^{6}$; Gavin S Chu ${ }^{4}$; Peter J Stafford ${ }^{4}$; Fernando S Schlindwein ${ }^{4}$; \\ G André $\mathrm{Ng}^{4}$; Tiago P Almeida ${ }^{1,4}$ \\ ${ }^{1}$ Aeronautics Institute of Technology, Brazil; ${ }^{2}$ Federal ABC University, Brazil; ${ }^{3}$ Karlsruhe Institute \\ of Technology, Germany; ${ }^{4}$ University of Leicester, UK; ${ }^{5}$ University of Trento, Italy; ${ }^{6}$ Eurac \\ Research, Italy
}

\begin{abstract}
Changes in atrial fibrillation cycle length $(A F-C L)$ are broadly used as a 'ground truth' to assess the effect of substrate modification during AF ablation. This work sought to optimize thresholds for changes in coronary sinus $C L(C S-C L)$ after local ablation using different atrial electrogram (AEG)-derived markers. 834 AEGs were collected from 11 patients undergoing persAF ablation. CS-CL was measured before and after each ablation point. Five AEG-derived markers were tested as classifiers for CS-CL changes: ICL (Biosense Webster), CFE-Mean (St. Jude Medical), Wave Similarity, Shannon Entropy and $A E G-C L$. The area under the receiver operating characteristic (AUROC) curve was used to assess the quality of classification for each marker. Maximum AUROC was found at threshold values between 9 and 14 ms in all markers, except for Shannon Entropy. The average AUROC of the five markers reached a maximum of 0.60 at a threshold value of $10 \mathrm{~ms}$. The $10 \mathrm{~ms}$ threshold is suggested as a starting setpoint for future studies seeking to identify $A F$ ablation targets based on an objective 'ground truth'.
\end{abstract}

\section{Introduction}

Atrial fibrillation (AF) is the most common sustained arrhythmia in the clinical practice and a leading cause of hospitalization and cardiovascular complications [1].

Catheter ablation is an effective treatment in the early phases of $\mathrm{AF}$, with pulmonary vein isolation (PVI) being the cornerstone for therapy [1]. Ablation, however, is less efficient when $\mathrm{AF}$ is sustained for longer periods of time (persistent AF, persAF). That is because sustained AF induces changes in the atrial tissue that, in turn, also participate in $\mathrm{AF}$ perpetuation, resulting in an intricate activation pattern, hindering the identification of the atrial regions that drive the arrhythmia [1]. Additionally, persAF is commonly driven by multiple mechanisms that may coexist, either simultaneously or intermittently, such as: multiple wavelets; ectopic foci; epi-endocardial dissociation; and reentry resulting from rotors [1]. These mechanisms differ from patient to patient, due to differences in anatomy, electrophysiology, and atrial substrate remodelling caused by sustained $\mathrm{AF}$, requiring patient-tailored ablation strategies [2].

Atrial substrate characterisation - and consequently, ablation target identification - is usually conducted considering numerical markers extracted from atrial electrograms (AEGs), each one tailored for the identification of different AF mechanisms [3].

Changes in atrial fibrillation cycle length (AF-CL) are broadly used as a 'ground truth' to assess the effect of substrate modification during AF ablation, and electrophysiological effects of the left atrium (LA) have been shown to be measurable from the coronary sinus (CS) [4], [5]. There is, however, no consensus on what extent of changes in AF-CL should be considered indicative of significant substrate modification, with different works reaching different values [1], [6], [7].

In this work, we sought to optimize thresholds for changes in CS-CL ( $\triangle$ CS-CL) after local ablation potentially leading to improved ablation strategies that can better identify targets to terminate persAF. The optimization was performed by assessing the classification performance of different AEG-derived markers, able to characterize the AF process, at varying $\triangle \mathrm{CS}-\mathrm{CL}$ threshold values.

\section{Methods and materials}

\subsection{Study population}

The population consisted of 11 patients ( 8 males; mean age $60.7 \pm 7.3$ years; history of AF $57.3 \pm 37.5$ months) referred to Glenfield Hospital (UK) for catheter ablation of persistent AF (persAF). 
3D LA geometry was created within Ensite NavX ${ }^{\text {тм }}$ (St.Jude Medical, St. Paul, Minnesota) using a deflectable, variable loop circular pulmonary vein (PV) mapping catheter (Inquiry Optima, St. Jude Medical). Sequential point-by-point bipolar AEGs were collected from different atrial regions.

In all cases, PVs were silent and all patients were in $\mathrm{AF}$ during signal collection. Sinus rhythm following substrateguided ablation was achieved in all cases.

\subsection{Signal Processing}

A total of 834 bipolar AEGs were sequentially recorded from different regions of the LA following PVI (5 s AEG duration; $1200 \mathrm{~Hz}$ sampling frequency). The AEGs were band-pass filtered between $30-300 \mathrm{~Hz}$ with a NavX system built in filter [8]. A $50 \mathrm{~Hz}$ Notch filter was applied to reduce power line noise. A stationary wavelet transform filter was implemented based on a previously described method to further reduce both baseline oscillations and high frequency noise [9].

The CS-CL was visually annotated as the mean duration of 10 activations in the AEGs recorded from the CS both before and after ablation clusters using LabSystem PRO (Boston Scientific, MA, USA).

\subsection{AEG-derived Markers}

Five AEG-derived markers were tested as classifiers for CS-CL changes:

ICL (CARTO system, Biosense Webster). The CARTO algorithm identifies peaks and troughs of bipolar AEGs whose absolute values are between an upper and a lower threshold and defines them as fractionated AEGs. The time intervals between successive peaks and troughs occurring within the voltage window are annotated. The complex intervals are marked within a time interval duration of 2.5 $\mathrm{s}$. The number of identified complex intervals is referred to as the interval confidence level (ICL) [8]. System default settings were used.

CFE-Mean (NavX system, St. Jude Medical). It is defined as the average time between activations marked by the NavX system. An activation is annotated in the AEG when i) it surpasses a certain peak-to-peak threshold; ii) it has a deflection duration within a certain threshold and; iii) it occurs after a refractory period following the previously marked activation [8]. System default settings were used.

Wave Similarity (WS). It measures the repetitiveness in AEG activation wave morphology. Local activation waves are identified and normalized, and pairs of waves are compared using the inner product. Those with an inner product above a certain threshold are deemed similar. WS is defined as the ratio of the similar activations and the total number of comparisons. The threshold was used as established in previous works [10].

Shannon Entropy (ShEn), It measures the "information content", or complexity, of a signal by writing it out as a discrete probability distribution of its samples $\left(p_{i}\right)$, as seen in equation 1 [11].

$$
\text { ShEn }=\sum_{i=1}^{N} p_{i} \ln \left(p_{i}\right)
$$

The signals were discretized into $\mathrm{N}=50$ equally spaced bins to obtain the probability distributions [12].

$A E G-C L$. It is the average time interval between subsequent activations in the AEG.

\subsection{Performance measurement}

AEGs collected at regions in which ablation resulted in CS-CL increase above a given threshold were labelled 'target', and those below the threshold were labelled 'nontarget'. Thresholds between 0 and $100 \mathrm{~ms}$ were investigated, and each value resulted in a different separation between the two classes (Figure 1A).

The area under the receiver operating characteristic (AUROC) curve was used to assess the quality of classification for each marker at each threshold value, and the optimal threshold to maximize AUROC for each marker was found (Figure 1B, 1C).

The average AUROC of the 5 studied markers was evaluated for each threshold value and used to propose a general optimal threshold for class separation. The optimal point of the ROC curve and corresponding accuracy, sensitivity, and specificity were computed for each marker at its optimal threshold, as well as at the proposed general threshold.

\section{Results}

Maximum AUROC was found at threshold values between 9 and $14 \mathrm{~ms}$ in all the markers, with the exception of ShEn (Table 1). Despite the low AUROC values, all the markers showed better performance than the random case.

The average AUROC of the five markers reached a maximum of 0.60 at threshold $10 \mathrm{~ms}$, which is one of the points of smallest variance (Figure 2). The lowest variance point was at threshold $15 \mathrm{~ms}$, with corresponding average AUROC $=0.58$.

All markers had negligible or small loss of performance when comparing their accuracy, sensitivity, and specificity at the respective optimal thresholds with those at the general threshold (Tables 2 and 3). This is in accordance with the low variance at the optimal average point, and is especially note-worthy for ShEn, whose optimal threshold was considerably larger than the others. 


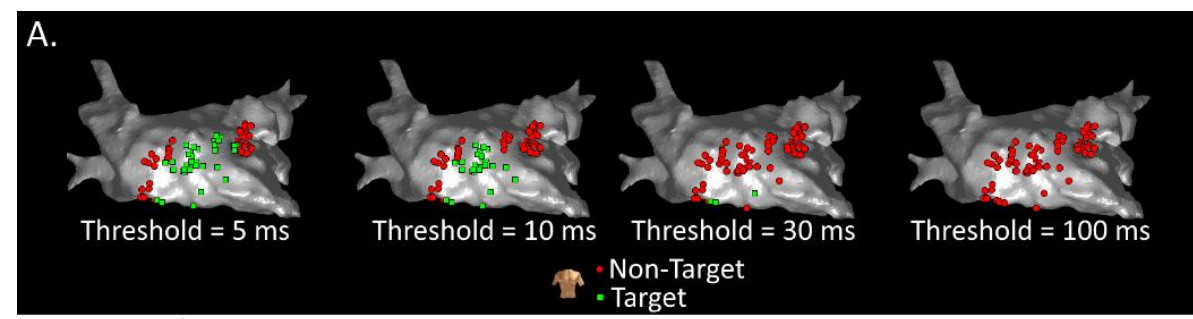

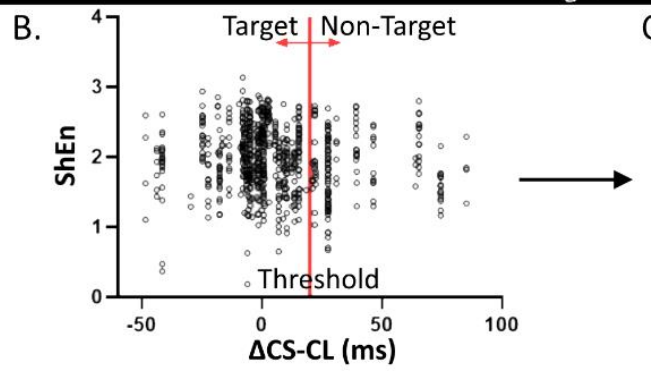

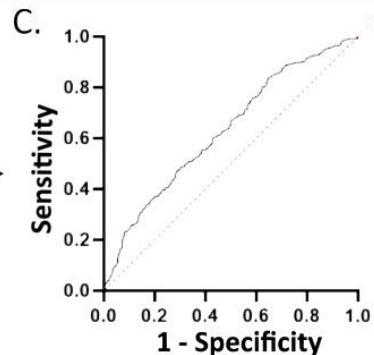

Figure 1: A. 3D map of a patient's LA showing the ablation points, labelled as 'target' and 'non-target', under different separation thresholds. B. Paired values of a marker (ShEn) vs CS-CL change ( $\triangle \mathrm{CS}-\mathrm{CL})$, and a threshold between 'target' and 'nontarget' labels. C. ROC curve corresponding to the given threshold.

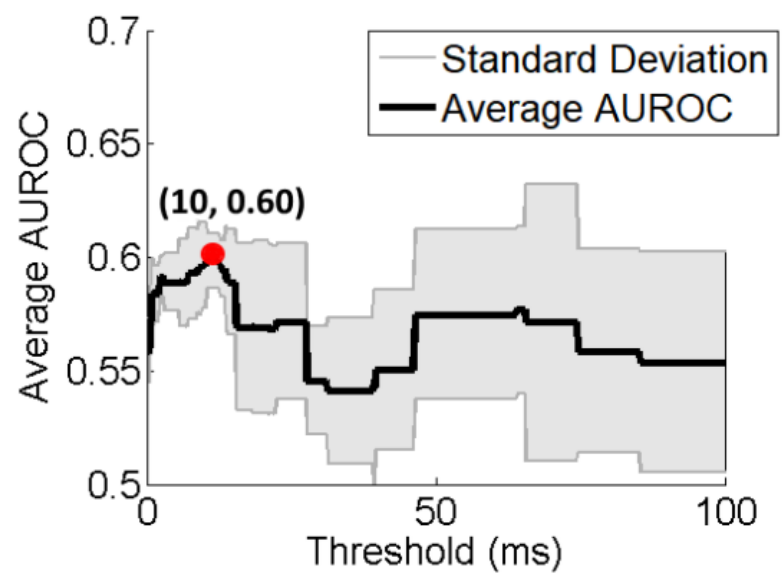

Figure 2: Average AUROC \pm standard deviation for each threshold value

Table 1. Highest AUROC and optimal threshold for each marker

\begin{tabular}{ccc}
\hline \hline Marker & $\begin{array}{c}\text { Highest } \\
\text { AUROC }\end{array}$ & $\begin{array}{c}\text { Optimal } \\
\text { Threshold }\end{array}$ \\
\hline ICL & 0.60 & 14 \\
CFE-Mean & 0.62 & 14 \\
WS & 0.62 & 9 \\
ShEn & 0.67 & 66 \\
AEG-CL & 0.62 & 10 \\
\hline \hline
\end{tabular}

\section{Discussion and Conclusion}

The $\triangle$ CS-CL threshold has a direct impact on the definition of atrial substrate and on the ground truth for AF perpetuation. Consequently, changes in $\triangle \mathrm{CS}$-CL threshold affect the two classes - target and non-target - which retrospective studies use to characterise AEGs and their features. Depending on the choice of $\triangle \mathrm{CS}-\mathrm{CL}$ threshold, ablation regions defined by such features might become too conservative and insufficient to terminate persAF, leaving room for $\mathrm{AF}$ recurrence. On the other hand, excessive ablation may burn healthy tissue, creating even more arrhythmogenic regions in the atrium, perpetuating even more the arrhythmia. In this work, we sought to find the 'middle ground' that could be the first step for more effective ablation strategy based on responses on the CSCL following substrate-guided ablation.

Previous works have set the threshold for AF-CL change that defines a significant ablation effect at $5 \mathrm{~ms}$ [1], $6 \mathrm{~ms}$ [7], and $30 \mathrm{~ms}$ [6]. This heterogeneity of results reinforces the importance of seeking an objective method to identify the threshold, which was pursued in this work by searching for threshold values that reconcile different markers - which reflect different mechanisms - into a unified threshold.

Table 2: Accuracy, sensitivity, and specificity of each marker using its respective optimal threshold

\begin{tabular}{cccc}
\hline \hline Marker & Accuracy & Sensitivity & Specificity \\
\hline ICL & 0.56 & 0.60 & 0.56 \\
CFE-Mean & 0.61 & 0.58 & 0.63 \\
WS & 0.59 & 0.60 & 0.58 \\
ShEn & 0.57 & 0.59 & 0.56 \\
AEG-CL & 0.63 & 0.60 & 0.66 \\
\hline \hline
\end{tabular}

Table 3: Accuracy, sensitivity, and specificity of each marker using the generalized threshold $(10 \mathrm{~ms})$

\begin{tabular}{cccc}
\hline \hline Marker & Accuracy & Sensitivity & Specificity \\
\hline
\end{tabular}




\begin{tabular}{cccc}
\hline ICL & 0.54 & 0.59 & 0.52 \\
CFE-Mean & 0.58 & 0.58 & 0.58 \\
WS & 0.56 & 0.58 & 0.53 \\
ShEn & 0.57 & 0.58 & 0.55 \\
AEG-CL & 0.63 & 0.60 & 0.66 \\
\hline \hline
\end{tabular}

Our results suggest that the studied markers have low but not negligible - capacity to individually identify the two $\triangle$ CS-CL-based classes, which could be related to the presence of mechanisms they're not capable of measuring, or simply to the fact that $\mathrm{AF}$ is too complex to be characterized by any single marker. Despite their different purposes and rationales, the markers mostly converged to a similar range of $\triangle \mathrm{CS}-\mathrm{CL}$ thresholds. The low AUROC variance around the optimal average point, as well as the small performance loss for all markers when using the general threshold, suggest that all markers perform relatively well in that range - even Shannon Entropy, whose optimal threshold was the most distant. This suggests that despite the low performance of the markers, there is a consistency of the $10 \mathrm{~ms}$ point, which may be potentially generalized to other markers or even other databases. This result can possibly be further strengthened by the introduction of novel markers that better correlate to $\Delta$ CS-CL.

A robust separation of AEGs into two classes ('targets', 'non-targets') based on $\triangle$ CS-CL can help to guide future studies seeking to identify persAF ablation targets - and in particular studies focusing on supervised classification, where the use of an objective electrophysiological 'ground truth' is crucial. The $10 \mathrm{~ms}$ threshold is suggested as a starting setpoint that unifies methods for different mechanisms to identify targets for persAF ablation in future studies.

\section{Acknowledgments}

The work reported in this paper was supported by the NIHR Leicester Biomedical Research Centre. ASB received a research grant from FAPESP (N. 2019/051921). DCS received support from FAPESP (2019/09512-0). XL received research grants from Medical Research Council UK (MRC DPFS ref: MR/S037306/1). FR received a research grant from Fondazione Cassa di Risparmio di Trento e Rovereto (2016.0273). TPA received research grants from São Paulo Research Foundation (FAPESP, Brazil, 2017/00319-8 and 2018/02251-4), from the British Heart Foundation (BHF Project Grant no. PG/18/33/33780), and the BHF Research Accelerator for Early Career Researcher.

\section{References}

[1] H. Calkins et al., '2017

HRS/EHRA/ECAS/APHRS/SOLAECE expert consensus statement on catheter and surgical ablation of atrial fibrillation', Heart Rhythm, vol. 14, no. 10, pp. e275e444, 2017, doi: 10.1016/j.hrthm.2017.05.012.

[2] Nattel Stanley, Burstein Brett, and Dobrev Dobromir, 'Atrial Remodeling and Atrial Fibrillation', Circ. Arrhythm. Electrophysiol., vol. 1, no. 1, pp. 62-73, Apr. 2008, doi: 10.1161/CIRCEP.107.754564.

[3] A. Verma et al., 'Substrate and Trigger Ablation for Reduction of Atrial Fibrillation (STAR AF): a randomized, multicentre, international trial', Eur. Heart $J$. , vol. 31, no. 11, pp. 1344-1356, Jun. 2010, doi: 10.1093/eurheartj/ehq041.

[4] M. Chauvin, D. C. Shah, M. Haïssaguerre, L. Marcellin, and C. Brechenmacher, 'The anatomic basis of connections between the coronary sinus musculature and the left atrium in humans', Circulation, vol. 101, no. 6, pp. 647-652, Feb. 2000, doi: 10.1161/01.cir.101.6.647.

[5] E. K. Heist, F. Chalhoub, C. Barrett, S. Danik, J. N. Ruskin, and M. Mansour, 'Predictors of atrial fibrillation termination and clinical success of catheter ablation of persistent atrial fibrillation', Am. J. Cardiol., vol. 110, no. 4, pp. 545-551, Aug. 2012, doi: 10.1016/j.amjcard.2012.04.028.

[6] M. Haïssaguerre et al., 'Changes in atrial fibrillation cycle length and inducibility during catheter ablation and their relation to outcome', Circulation, vol. 109, no. 24, pp. 3007-3013, Jun. 2004, doi: 10.1161/01.CIR.0000130645.95357.97.

[7] Y. Takahashi et al., 'Characterization of electrograms associated with termination of chronic atrial fibrillation by catheter ablation', J. Am. Coll. Cardiol., vol. 51, no. 10, pp. 1003-1010, Mar. 2008, doi: 10.1016/j.jacc.2007.10.056.

[8] T. P. Almeida et al., 'Minimizing discordances in automated classification of fractionated electrograms in human persistent atrial fibrillation', Med. Biol. Eng. Comput., vol. 54, no. 11, pp. 1695-1706, Nov. 2016, doi: 10.1007/s11517-016-1456-2.

[9] C. Schilling, 'Analysis of Atrial Electrograms', KIT Sci. Publ., 2012.

[10] F. Ravelli et al., 'Anatomic localization of rapid repetitive sources in persistent atrial fibrillation: fusion of biatrial CT images with wave similarity/cycle length maps.', JACC Cardiovasc. Imaging, vol 5, no. 12, pp. 1211-1220, 2012, doi: 10.1016/j.jcmg.2012.07.016.

[11] C. E. Shannon, 'A Mathematical Theory of Communication', p. 55.

[12] T. P. Almeida et al., 'Atrial electrogram fractionation distribution before and after pulmonary vein isolation in human persistent atrial fibrillation - a retrospective multivariate statistical analysis', Front. Physiol., vol. 8, 2017, doi: 10.3389/fphys.2017.00589.

Address for correspondence:

Arthur Santos Bezerra

Aeronautics Institute of Technology

Praça Marechal Eduardo Gomes, 50 - São José dos Campos

São Paulo, Brazil, 12228-900

arthursantosbezerra@gmail.com 